\section{Reprodutibilidade de questionário semiquantitativo de freqüência alimentar elaborado para adolescentes da Região Metropolitana do Rio de Janeiro, Brasil}

\author{
Reliability of a semi-quantitative food frequency \\ questionnaire designed for adolescents from the \\ Rio de Janeiro Metropolitan Area, Brazil
}

Marina Campos Araújo 1

Daniele Mendonça Ferreira 2 Rosangela Alves Pereira 1

\footnotetext{
1 Instituto de Nutrição Josué de Castro, Universidade Federal do Rio de Janeiro, Brasil.

2 Faculdade de Nutrição Universidade Federal

Fluminense, Brasil.

Correspondência R. A. Pereira

Instituto de Nutrição Josué de Castro, Centro de Ciências da Saúde, Universidade Federal do Rio de Janeiro. Av. Carlos Chagas Filho 373, Edifício do Centro de Ciências da Saúde, Bloco J, 2o andar, Rio de Janeiro, RJ 21941-902, Brasil. roapereira@ufrj.br rpereira@pobox.com
}

\begin{abstract}
A test-retest study was carried out to assess the reproducibility of a semi-quantitative food frequency questionnaire (SFFQ) designed for adolescents. The study group included 108 individuals. The mean interval between the two interviews was 20 days. Three days of food records from 430 adolescents were used to define the items and portions in the SFFQ. Intraclass correlation coefficients (ICC) were estimated to assess the agreement in food item reports. The agreement for energy and nutrient intake was assessed by Pearson's correlation coefficients, weighted kappa (agreement in quartile classification), and the Bland-Altman method. Data for nutrients were adjusted according to energy intake. Mean ICC was 0.54. The categorization in the same and in the adjacent quartile for data adjusted for energy intake ranged from $72 \%$ to $86 \%$ (mean $=80 \%$ ). According to the Bland-Altman method, mean agreements ranged from $77 \%$ to $104 \%$ and were independent of the intake variation, except for vitamin A. Pearson's correlation coefficients ranged from 0.40 to 0.61 ( mean $=0.52$ ). The SFFQ showed acceptable reliability to assess food, energy, and nutrient intake by adolescents in the study area.
\end{abstract}

Food Consumption; Questionnaires; Adolescent
Introdução

A investigação sobre consumo de alimentos é tema de grande importância para a saúde pública, pois contribui para fundamentar políticas de alimentação e nutrição e a elaboração de guias alimentares e recomendações. Além disso, estudos epidemiológicos que analisam a ingestão alimentar têm fornecido evidências sobre a importância da dieta na etiologia de diversas doenças 1 .

Entretanto, Beaton 2 assinala que a ingestão dietética não é estimada sem erro. A natureza e a magnitude do erro dependem da metodologia escolhida para a coleta dos dados, e de diversos outros fatores, como as características dos indivíduos analisados. Entre os métodos mais utilizados para avaliação do consumo alimentar de grupos populacionais, o questionário de freqüência alimentar (QFA) tem sido considerado um método prático e informativo de avaliação da ingestão dietética usual 3 .

Basicamente, o QFA possui dois componentes: uma lista de alimentos e uma série de categorias de freqüência de consumo. O respondente deve escolher a freqüência que descreve o seu consumo usual de cada item no período de referência do questionário. Opcionalmente, podem ser incluídas porções padronizadas no questionário permitindo que a quantidade consumida usualmente seja estimada. A principal característica do QFA é a possibilidade de categorizar os indivíduos segundo gradientes de consumo e, 
dessa forma, propiciar a estimativa da associação entre dieta e desenvolvimento de enfermidades. Entre as vantagens do QFA estão a facilidade de aplicação e análise dos dados, e o menor custo em comparação com os outros métodos 4,5.

A importância da determinação da reprodutibilidade e validade de QFAs está relacionada ao fato de que estes devem ser específicos para as populações para os quais foram elaborados, uma vez que a lista dos alimentos deve ser baseada no consumo habitual dessa população 6 . A reprodutibilidade ou confiabilidade mede a consistência e a precisão do QFA na avaliação de um mesmo indivíduo em diferentes pontos no tempo, ou seja, indica se o instrumento é capaz de fornecer resultados semelhantes quando aplicado repetidas vezes $7,8,9$.

Este trabalho apresenta os resultados da reprodutibilidade de questionário semiquantitativo de freqüência alimentar (QSFA) elaborado para estimar o consumo alimentar de adolescentes da Região Metropolitana do Rio de Janeiro, Brasil.

\section{Material e métodos}

\section{Desenvolvimento do QSFA}

O QSFA utilizado no presente estudo foi construído com base em dados obtidos em investigação sobre fatores de risco para doenças cardiovasculares realizado em 2003, em amostra probabilística de adolescentes de 12 a 19 anos de idade, matriculados em escolas estaduais de Niterói, Rio de Janeiro. Para a definição da lista de alimentos tomaram-se por base as informações dos 430 adolescentes que completaram três dias de registro alimentar não consecutivos, sendo dois dias úteis e um dia de final de semana.

Na seleção dos alimentos que iriam compor a lista, os itens relatados nos registros foram organizados em ordem decrescente de freqüência de citação e foram incluídos aqueles referidos pelo menos 15 vezes, quando considerados isoladamente ou em combinações de produtos similares; por exemplo, oito preparações para compor a categoria carne de boi: bife, carne assada, carne moída, carne ensopada, estrogonofe de filé mignon, almôndega, carne, espetinho de carne.

Nos registros analisados foram citados 308 itens alimentares; destes, 245 foram mantidos no QSFA, compondo uma lista de noventa itens. Dos 63 alimentos mencionados menos de 15 vezes nos registros, cinco foram mantidos no QSFA: peixe enlatado, cerveja, vinho, bebidas destiladas e refrigerantes de baixa caloria, considerando que mesmo sendo pouco referidos, alguns itens podem ter capacidade de discriminar o consumo alimentar ou podem apontar tendências na modificação dos hábitos alimentares de grupos da população.

Adicionalmente, a metodologia proposta por Block et al. 10 foi empregada para avaliar a consistência da lista de alimentos estabelecida para o QSFA e confirmar a contribuição de cada item alimentar incluído no QSFA para o consumo alimentar da população estudada. Assim, estimouse a contribuição percentual de cada um dos alimentos listados nos registros para o consumo total de energia, macronutrientes, colesterol, vitaminas A e C, ferro e cálcio e elaborou-se uma lista com os alimentos que contribuíam com $95 \%$ do consumo total de energia e dos nutrientes considerados.

Ao cotejarem-se as duas listas, aquela elaborada por ordem de citação e a desenvolvida por contribuição percentual ao consumo de energia e nutrientes, observou-se que a primeira contemplava todos os itens que apresentaram contribuição substancial para o consumo dos nutrientes investigados.

Para determinar porções de referência dos itens alimentares incluídos no QSFA, utilizaramse as "porções naturais" ou "típicas" de alimentos: como pão francês, banana, laranja, maçã, ovo, cachorro-quente, hambúrguer; enquanto que para outros alimentos (como arroz, feijão, carnes etc.) as porções de referência foram as mais freqüentemente referidas ("porções modais") nos registros do estudo de base. Também foram consideradas as medidas caseiras empregadas para servir alimentos mais citadas em enquete sobre utensílios domésticos mais utilizados para servir alimentos, desenvolvida entre 35 homens e 35 mulheres de 19 a 67 anos de idade. Nessa enquete, os indivíduos foram expostos a 45 utensílios entre os quais identificavam aqueles que usavam para servir 22 tipos de alimentos 11 .

A definição das opções de freqüência baseouse nas utilizadas em QSFA desenvolvido para adultos no Rio de Janeiro 12 e naquelas apresentadas no questionário para crianças e adolescentes desenvolvido nos Estados Unidos 13. Organizaramse as opções de freqüência de forma diferenciada para os distintos itens do questionário, tendo em vista facilitar a resposta às questões, uma vez que a freqüência do consumo é usualmente distinta para os diferentes alimentos e que poucos alimentos são consumidos mais de uma vez ao dia, evitando o super-relato no consumo desses itens 14. Para 14 itens alimentares que figuram entre os alimentos mais consumidos entre adolescentes do Rio de Janeiro em estudo realizado em 19951996 por Andrade et al. 15 - por exemplo: leite, café, pão francês, arroz, feijão, entre outros - foram 
determinadas oito opções de freqüência, que variaram de "menos de uma vez por mês ou nunca" a "quatro ou mais vezes ao dia". Para outros 18 alimentos (ex.: achocolatado em pó, iogurte, pão doce, queijo, laranja, ovo etc.) as opções de freqüência variaram de "menos de uma vez por mês ou nunca" a "duas ou mais vezes por dia". E para 58 itens, as cinco opções de freqüências definidas variaram de "menos de uma vez por mês ou nunca" a "cinco ou mais vezes por semana”. Após pré-teste, optou-se por formatar o questionário no formato vertical.

Foram integradas ao QSFA questões sobre hábitos alimentares abordando informações sobre o uso de açúcar, adoçante, azeite de oliva, tipo de leite usualmente consumido, consumo de frituras, pele de frango, gordura aparente das carnes, local de refeições, hábito de "beliscar" entre as refeições e uso de suplementos vitamínicos.

O período de referência utilizado para estimar a freqüência do consumo de alimentos foram os seis meses precedentes à entrevista.

\section{Grupo investigado}

Na seleção dos adolescentes para este estudo procurou-se incluir indivíduos de diferentes áreas geográficas da Região Metropolitana do Rio de Janeiro. Foram investigados 114 adolescentes; sendo 82 estudantes de uma escola estadual de Niterói e 32 adolescentes residentes no 2o Distrito do Município de Duque de Caxias. Para selecionar os alunos da escola, distribuíram-se Termos de Consentimento Livre e Esclarecido para todos os alunos de 6ạ e 7a séries do Ensino Fundamental, dos turnos da manhã e tarde (totalizando 140 alunos) e foram incluídos no estudo todos aqueles que retornaram o termo de consentimento assinado pelo responsável e que completaram as duas aplicações do QSFA.

Foram selecionados 58 adolescentes residentes em Duque de Caxias investigados na pesquisa de base populacional Avaliação do Estado Nutricional, Hábitos Alimentares e Insegurança Alimentar no Município de Duque de Caxias, Rio de Janeiro: Desenvolvimento de um Instrumento Simplificado para Avaliação de Consumo Alimentar Saudável 16. Assim, foram selecionados 58 adolescentes e 32 (55\%) completaram o questionário duas vezes.

\section{Coleta de dados}

Desenvolveu-se estudo de teste-reteste, que consistiu em duas aplicações do QSFA (QSFA 1 : primeira aplicação e QSFA $_{2}$ : segunda aplicação) prevendo-se um intervalo de três semanas entre as mesmas. O QSFA foi aplicado em entrevistas individuais que duravam, em média, 15-20 minutos. As entrevistas foram realizadas por nutricionistas e acadêmicos de nutrição, previamente treinados. Foi elaborado um manual de campo para a padronização dos procedimentos. No decorrer do estudo foram realizadas sessões de re-treinamento, objetivando o aperfeiçoamento da entrevista e a minimização da variabilidade inter-entrevistador. Os dados foram coletados entre maio e julho de 2005 em Niterói e entre janeiro e abril de 2006 em Duque de Caxias.

\section{Análises estatísticas}

A freqüência de consumo relatada para cada item foi transformada em freqüência diária. Para tanto, considerou-se a opção de freqüência "uma vez ao dia" como freqüência diária igual a 1. As demais opções foram relacionadas proporcionalmente com a unidade. Assim, para os itens referidos como consumidos duas a três vezes ao dia, a freqüência diária era 2,5 (2+3/2); para itens consumidos duas a quatro vezes por semana a freqüência diária era 0,43 $[(2+4 / 2) / 7]$. Foram estimados as médias da freqüência diária de consumo de cada item alimentar do questionário e os respectivos intervalos de confiança para as duas aplicações do QSFA. Estimaram-se os coeficientes de correlação intraclasse para o consumo de cada item entre as duas aplicações do QSFA.

$\mathrm{O}$ consumo de energia e nutrientes foi estimado utilizando-se o Programa de Apoio à Nutrição - NutWin (Departamento de Informática em Saúde, Universidade de São Paulo, Brasil). Os valores de consumo de energia e nutrientes foram transformados em logaritmos dado que as duas distribuições eram assimétricas. O consumo estimado de nutrientes foi ajustado pelo consumo energético pelo método da densidade energética.

O consumo de energia e nutrientes foi categorizado em quartis em ambas as aplicações do questionário e estimou-se a concordância desta categorização com o uso da estatística kappa ponderado. Adicionalmente, avaliou-se a proporção de adolescentes categorizados no mesmo quartil de consumo nas duas aplicações do QSFA (concordância exata); os categorizados nos quartis adjacentes (concordância adjacente) e os adolescentes categorizados nos quartis distantes e nos quartis opostos (discordância).

As médias do consumo de energia e nutrientes e as diferenças entre os questionários foram analisadas segundo a metodologia proposta por Bland \& Altman 17. Para verificar se a concordância entre as duas aplicações do questionário variou com a magnitude do consumo, desenvolveu-se procedimento de regressão linear, tendo 
como variável dependente a diferença entre as duas aplicações do QFCA e variável independente a média das duas aplicações. Estimaram-se os coeficientes de correlação de Pearson para as médias de consumo de energia e nutrientes nas duas aplicações do QSFA.

As análises estatísticas foram realizadas no pacote estatístico SPSS versão 13.0 (SPSS Inc., Chicago, Estados Unidos). Para a avaliação da significância estatística foi considerado o valor de $\mathrm{p}<0,05$.

\section{Aspectos éticos}

Esta pesquisa foi aprovada pelo Comitê de Ética em Pesquisa do Instituto de Medicina Social da Universidade Estadual do Rio do Janeiro em 13 de janeiro de 2005. A participação na pesquisa foi condicionada à assinatura do Termo de Consentimento Livre e Esclarecido por responsável ou pelo indivíduo com idade igual ou maior de 18 anos.

\section{Resultados}

Foram entrevistados 114 adolescentes (32 meninos e 82 meninas). Foram excluídos seis questionários que apresentaram consumo energético inferior a $500 \mathrm{kcal}$ ou maior que $6.000 \mathrm{kcal}$, que podem ser considerados consumo usuais pouco prováveis 15 , sendo analisados dados de 108 adolescentes, dos quais $73 \%(n=79)$ eram meninas e $27 \%(n=29)$ meninos com idades variando de 11 a 19 anos (média = 14,6 anos; desvio-padrão - DP =1,9 anos). Na categorização por faixa etária 69 adolescentes (64\%) tinham idades entre 11 e 14 anos e 39 adolescentes (36\%) entre 15 e 19 anos de idade. O intervalo médio entre as duas aplicações do QSFA foi de vinte dias, variando de 11 a 67 dias.

Os coeficientes de correlação intraclasse para a freqüência de consumo diário dos itens alimentares variaram de -0,09 (batata cozida) a 0,91 (pepino), sendo a média $=0,54$. Para 66 itens alimentares foram estimados coeficientes iguais ou maiores que 0,5 e para 85 alimentos o coeficiente de correlação intraclasse foi estatisticamente significante. Para o relato de consumo de creme de leite, sopa industrializada, doce de leite, batata cozida e refrigerante light, o coeficiente de correlação intraclasse não foi estatisticamente significativo (dados não mostrados).

A análise da concordância da classificação em quartis de energia e nutrientes do QSFA $_{1}$ e $\mathrm{QSFA}_{2}$ resultou em kappa bruto significativo $(\mathrm{p}<$ 0,0001 ) para todas as variáveis analisadas, que variaram de 0,46 (colesterol) a 0,62 (cálcio), média $=0,50$ (dados não mostrados).
Para o consumo de nutrientes ajustado para o consumo de energia observou-se redução dos valores do kappa ponderado, que variaram de 0,33 (colesterol) a 0,54 (vitamina A), média $=0,46$, exceto para fibra que manteve o mesmo valor. Todas as variáveis mantiveram sua significância estatística $(\mathrm{p}<0,0001)$.

A proporção média de adolescentes categorizados no mesmo quartil de consumo de energia e nutrientes ajustados para ingestão energética (concordância exata) foi de $41 \%$ e em quartis opostos (discordância) foi de $20 \%$, para o consumo nas duas aplicações do QSFA. A concordância exata somada à adjacente foi em média de $80 \%$ entre os adolescentes categorizados (Tabela 1).

Considerando a metodologia de Bland \& Altman 17 , observou-se que as concordâncias médias do consumo de energia e nutrientes brutos entre as duas aplicações do QSFA variaram de $70 \%$ (vitamina C) a $98 \%$ (fibra). Para facilitar a comparação com outros estudos, calculou-se o coeficiente de correlação de Pearson entre as estimativas do consumo de energia e nutrientes de cada questionário. Os coeficientes de correlação brutos foram estatisticamente significantes para energia e todos nutrientes investigados variando de 0,48 (colesterol) a 0,71 (cálcio), valor médio = 0,60 (Tabela 2).

Para dados ajustados para consumo de energia, a concordância segundo o método de Bland \& Altman 17 manteve-se mais próxima da concordância ideal (100\%) para os nutrientes analisados. Foi observada redução nos coeficientes de correlação de Pearson quando os nutrientes foram ajustados para energia (média $=0,52$ ), exceto para o consumo de zinco, no qual o coeficiente de correlação de Pearson aumentou e para o consumo de lipídio que não se alterou. Observaram-se coeficientes de correlação estatisticamente significativos para todos os nutrientes analisados, exceto para o consumo de vitamina C (Tabela 2).

Para todos os nutrientes, exceto para vitamina $\mathrm{A}$, as análises de regressão linear demonstraram que a concordância foi independente da magnitude do consumo, como exemplificado para o consumo de colesterol bruto e ajustado (Figura 1). No caso da vitamina A, o coeficiente de regressão linear foi estatisticamente significativo tanto para a análise bruta como ajustada, evidenciando que a concordância para o consumo de vitamina A variou com a magnitude do consumo (Figura 2).

\section{Discussão}

A avaliação do consumo alimentar na fase da adolescência ganhou ênfase nos últimos anos frente 
Kappa ponderado e concordância do consumo de energia e nutrientes ajustados para o consumo energético entre as duas aplicações do questionário semiquantitativo de freqüência alimentar (QSFA) segundo a categorização em quartis. Região Metropolitana do Rio de Janeiro, Brasil, 2005-2006.

\begin{tabular}{|c|c|c|c|c|}
\hline $\begin{array}{l}\text { Energia/ } \\
\text { Nutrientes } \\
\text { ajustados }\end{array}$ & $\begin{array}{c}\text { Kappa } \\
\text { ponderado * }\end{array}$ & $\begin{array}{c}\text { Concordância } \\
\text { exata (\%) }\end{array}$ & $\begin{array}{c}\text { Concordância } \\
\text { exata + } \\
\text { adjacente (\%) }\end{array}$ & $\begin{array}{c}\text { Discordância } \\
\text { (\%) }\end{array}$ \\
\hline Energia (kcal) & 0,57 & 44 & 86 & 14 \\
\hline Proteína (g) & 0,49 & 41 & 83 & 17 \\
\hline Glicídio (g) & 0,39 & 43 & 72 & 28 \\
\hline Lipídio (g) & 0,50 & 40 & 83 & 17 \\
\hline Colesterol (mg) & 0,33 & 34 & 75 & 25 \\
\hline Fibra (g) & 0,53 & 50 & 83 & 17 \\
\hline Cálcio (mg) & 0,44 & 38 & 76 & 24 \\
\hline Ferro (mg) & 0,40 & 40 & 78 & 22 \\
\hline Zinco (mg) & 0,41 & 40 & 78 & 22 \\
\hline Fósforo (mg) & 0,42 & 37 & 81 & 19 \\
\hline Ácido fólico (mg) & 0,50 & 44 & 81 & 19 \\
\hline Vitamina A (UI) & 0,54 & 44 & 85 & 15 \\
\hline Vitamina C (mcg) & 0,47 & 40 & 82 & 18 \\
\hline Média & 0,46 & 41 & 80 & 20 \\
\hline
\end{tabular}

$\star p<0,001$

ao reconhecimento de que diversas enfermidades crônicas não-transmissíveis associam-se ao consumo alimentar praticado na adolescência 18,19. O QFA vem sendo considerado o método de escolha em estudos epidemiológicos que envolvem grande número de indivíduos e os dados sobre consumo alimentar obtidos a partir desse instrumento são correlacionados com a ingestão habitual de alimentos 20

Instrumentos de avaliação do consumo de alimentos desenvolvidos para adolescentes ainda são escassos no Brasil, entretanto sua aplicação é útil para o acompanhamento de avaliação do consumo e identificação de modificações no comportamento alimentar. No Rio de Janeiro, apenas um QFA foi validado para estimar o consumo da população adulta 12 .

Este estudo tem como principal contribuição a avaliação da confiabilidade de um novo questionário de freqüência de consumo alimentar desenhado para investigar o consumo de alimentos de adolescentes do Rio de Janeiro, permitindo assim analisar se o instrumento foi capaz de produzir resultados similares quando aplicado mais de uma vez.

A reprodutibilidade do QSFA foi avaliada pela comparação de duas aplicações do questionário com intervalo médio de vinte dias, considerado curto o suficiente para minimizar as chances de mudanças reais no consumo de alimentos, embora longo o bastante para evitar que as respostas no primeiro relato fossem repetidas com facilidade 21 . O intervalo de tempo entre as aplicações do QSFA variou de 11 a 67 dias. Essa amplitude é justificada pelo período das férias de julho que ocorreu durante a coleta dos dados dos estudantes de Niterói. O limite superior de 67 dias foi observado em apenas um dos estudantes. Por outro lado, a iminência das férias de final de ano fez com que cinco adolescentes fossem entrevistados pela segunda vez em período menor do que o previsto. Assim, para seis adolescentes (5\%) o intervalo de tempo entre o teste e o reteste foi diferenciado. Análises excluindo esses adolescentes não mostraram resultados diferenciados, por isso, conclui-se que a variação no intervalo de tempo não teve efeito importante sobre a reprodutibilidade do questionário.

Burley et al. 21 sugerem um intervalo de 15 a 45 dias entre o teste e o reteste em estudos de confiabilidade de QFA. Similarmente ao empregado neste estudo, outros trabalhos que avaliaram a confiabilidade do QFA para adolescentes utilizaram intervalo de tempo de aproximadamente um mês entre as aplicações do questionário 22,23,24,25.

Poucos estudos analisaram a reprodutibilidadedeQFA para itens alimentares. Hansson et al. 26, 
Concordância e correlação entre consumo de energia e nutrientes brutos e ajustados para o consumo energético em estudo de reprodutibilidade de questionário semiquantitativo de freqüência alimentar (QSFA) para adolescentes. Região Metropolitana do Rio de Janeiro, Brasil, $2005-2006$.

\begin{tabular}{|c|c|c|c|c|c|}
\hline & $\begin{array}{l}\text { Concordância } \\
\text { média (\%) }\end{array}$ & IC95\% ** & 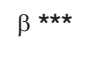 & Valor de p \# & $r \#$ \\
\hline \multicolumn{6}{|c|}{ Consumo de energia/nutrientes bruto * } \\
\hline Energia (kcal) & 91 & $52-160$ & 0,087 & 0,328 & 0,67 \\
\hline Proteína (g) & 93 & 53-162 & 0,018 & 0,854 & 0,62 \\
\hline Glicídio (g) & 90 & $51-160$ & 0,091 & 0,352 & 0,60 \\
\hline Lipídio (g) & 92 & 44-193 & 0,107 & 0,310 & 0,55 \\
\hline Colesterol (mg) & 90 & $32-247$ & 0,023 & 0,846 & 0,48 \\
\hline Fibra (g) & 98 & $43-224$ & 0,071 & 0,442 & 0,63 \\
\hline Cálcio (mg) & 84 & $42-169$ & 0,000 & 0,995 & 0,71 \\
\hline Ferro (mg) & 91 & $48-173$ & 0,056 & 0,608 & 0,53 \\
\hline Zinco (mg) & 94 & $52-172$ & 0,076 & 0,454 & 0,58 \\
\hline Fósforo (mg) & 93 & $54-158$ & 0,084 & 0,328 & 0,67 \\
\hline Ácido fólico (mg) & 94 & $37-236$ & 0,122 & 0,211 & 0,60 \\
\hline Vitamina A (UI) & 80 & $17-378$ & 0,250 & 0,012 & 0,59 \\
\hline Vitamina C (mcg) & 70 & $4-1268$ & 0,143 & 0,192 & 0,52 \\
\hline \multicolumn{6}{|c|}{ Consumo de nutrientes ajustado * } \\
\hline Proteína (g) & 102 & $78-133$ & 0,047 & 0,662 & 0,53 \\
\hline Glicídio (g) & 99 & $84-117$ & 0,056 & 0,644 & 0,44 \\
\hline Lipídio (g) & 101 & $72-141$ & 0,011 & 0,916 & 0,55 \\
\hline Colesterol (mg) & 98 & $41-233$ & $-0,003$ & 0,978 & 0,40 \\
\hline Fibra (g) & 107 & $53-217$ & $-0,075$ & 0,437 & 0,61 \\
\hline Cálcio (mg) & 93 & $55-158$ & 0,100 & 0,327 & 0,57 \\
\hline Ferro (mg) & 100 & $73-136$ & $-0,062$ & 0,611 & 0,43 \\
\hline Zinco (mg) & 104 & $74-145$ & $-0,106$ & 0,290 & 0,58 \\
\hline Fósforo (mg) & 102 & $75-138$ & 0,095 & 0,350 & 0,57 \\
\hline Ácido fólico (mg) & 103 & $48-224$ & 0,062 & 0,538 & 0,57 \\
\hline Vitamina A (UI) & 87 & $20-390$ & 0,219 & 0,045 & 0,52 \\
\hline Vitamina C (mcg) & 77 & $5-1264$ & 0,118 & 0,292 & $0,50 \# \# \#$ \\
\hline
\end{tabular}

* Análise foi realizada para os dados transformados em logaritmo;

** Limites de concordância = média das diferenças $\pm 1,96 \times$ desvio-padrão da distribuição das diferenças;

*** Coeficientes obtidos em análise de regressão linear entre as médias (variável independente) e as diferenças (variável dependente) nas duas aplicações do questionário:

\# Significância estatística do $\beta$;

\#\# Coeficiente de correlação de Pearson;

$\# \#$ \# $>0,05$.

em estudo de reprodutibilidade de QFA para adultos, observaram coeficientes de correlação de Spearman para itens alimentares variando de $-0,03$ a 0,83 (média $=0,57$ ), semelhante ao observado neste estudo. Matthys et al. 22 avaliando confiabilidade de QFA para adolescentes estimaram coeficientes de correlação de Spearman para grupos de alimentos variando de 0,27 a 0,87 (média $=0,62$ ). Cullen \& Zakeri 24 também avaliaram a confiabilidade de QFA para itens alimentares e estimaram coeficientes de correlação de Spearman variando entre 0,37 a 0,67 . Adicionalmente, a reprodutibilidade de um QFA para adolescentes foi testada para dez grupos de alimentos e encontraram-se coeficientes de correlação de Pearson que variaram de 0,46 a 0,85 quando o QFA foi aplicado com um intervalo de 48 horas. Quando o mesmo questionário foi aplicado com intervalo de duas semanas, sua análise proporcionou coeficientes que variaram de 0,05 a 0,82 27 .

Para cinco itens do questionário foram observados coeficientes de correlação não significativos: creme de leite, sopa industrializada, doce de leite, batata cozida e refrigerante light. Hansson 
Figura 1

Concordância média e limites de concordância superior e inferior entre o consumo de colesterol (mg) bruto e ajustado para energia estimado nos questionários semiquantitativos de freqüência alimentar (QSFA ${ }_{1}$ e QSFA $)_{2}$ *. Região Metropolitana do Rio de Janeiro, Brasil, 2005-2006.

1a) Colesterol (bruto)

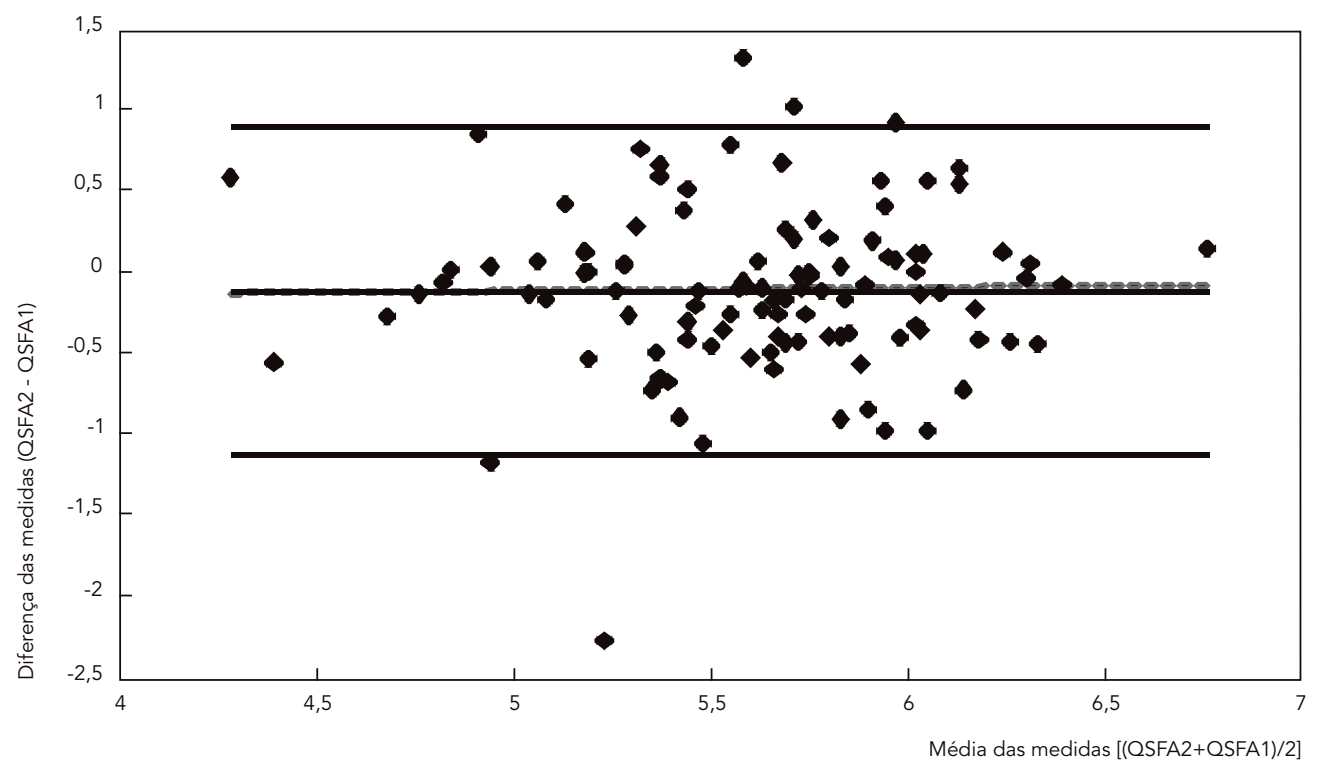

1b) Colesterol (ajustado)

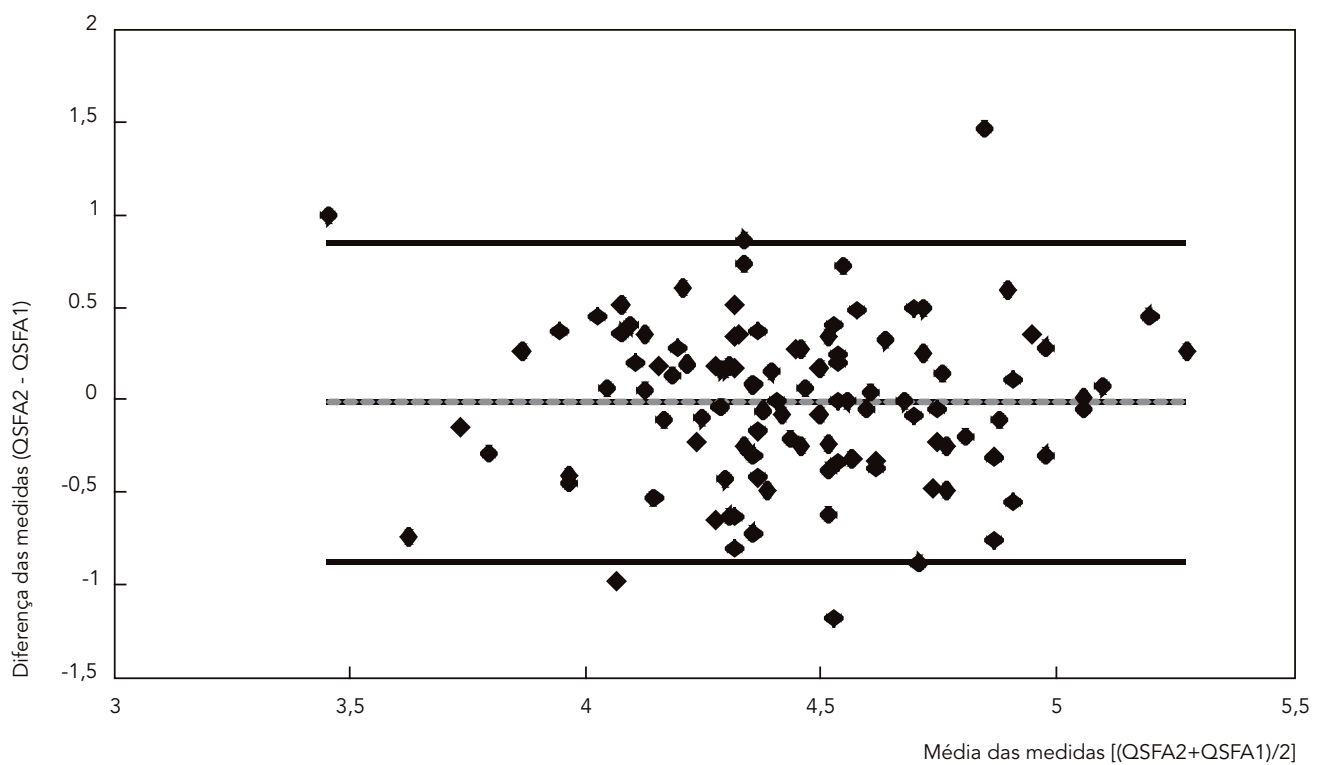

* Dados transformados em logaritmo. 


\section{Figura 2}

Concordância média e limites de concordância superior e inferior entre o consumo de vitamina A (UI) bruto e ajustado para energia estimado nos questionários semiquantitativos de freqüência alimentar (QSFA 1 e QSFA $)$ *. Região Metropolitana do Rio de Janeiro, Brasil, 2005-2006

2a) Vitamina A (bruto)

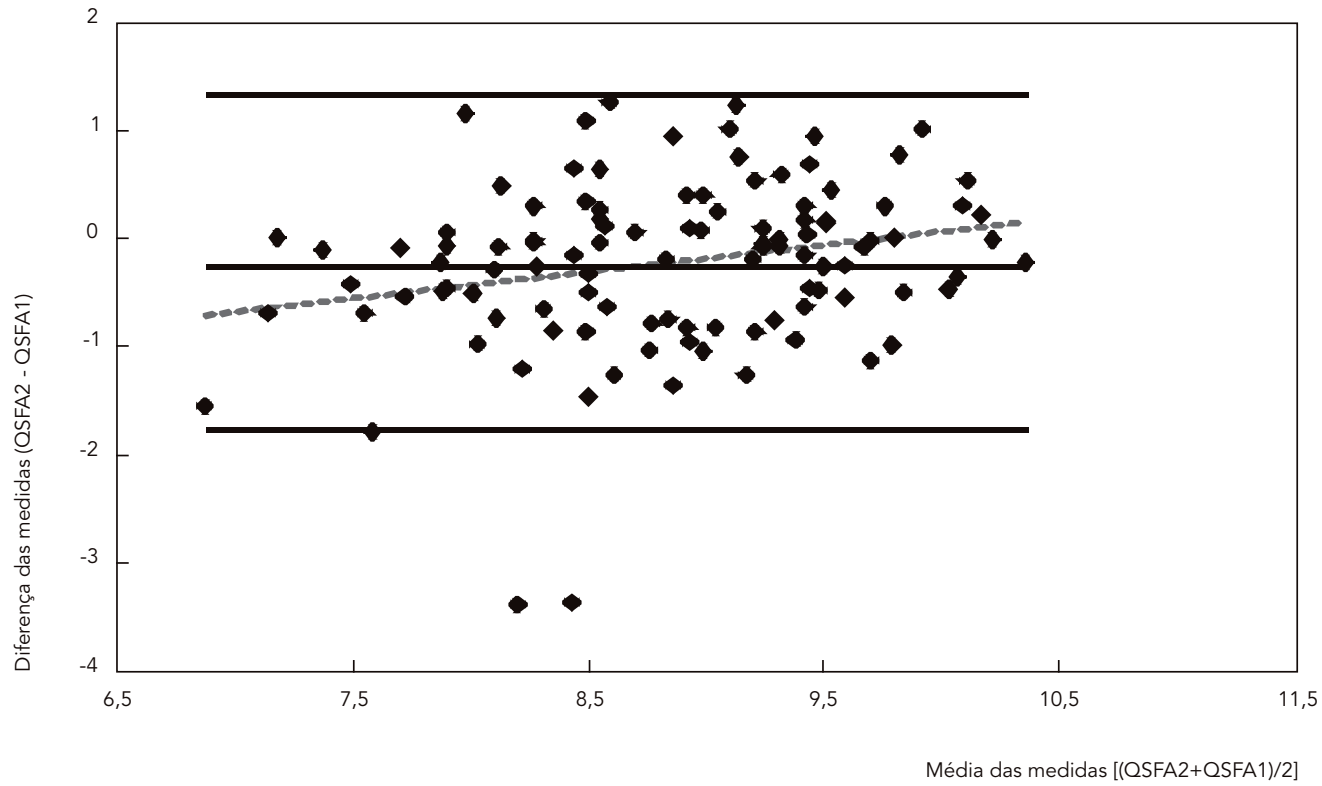

2b) Vitamina A (ajustado)

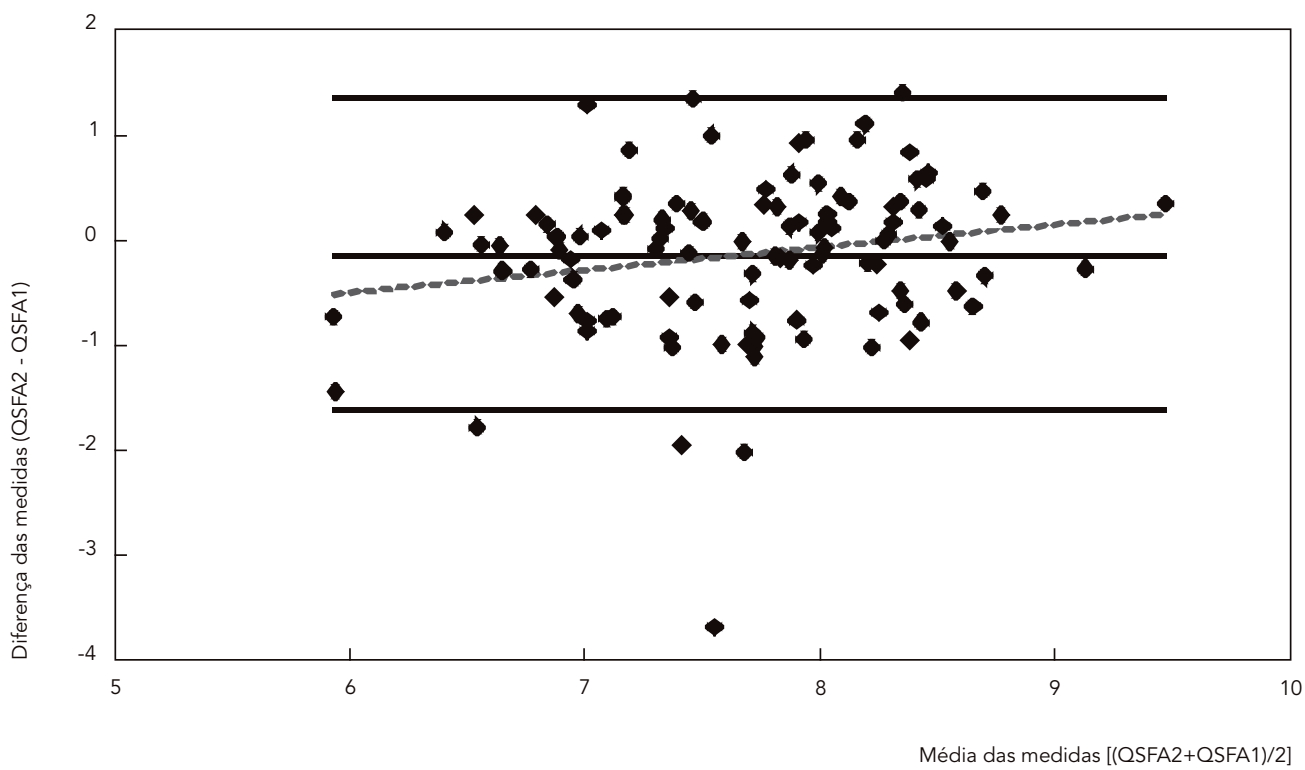

* Dados transformados em logaritmo. 
et al. 26 observaram reprodutibilidade média baixa para itens alimentares raramente consumidos e com uma distribuição assimétrica de consumo, ou seja, alimentos raramente consumidos por grande parte da população e com consumo aumentado para pequena parcela de indivíduos. Tal observação poderia explicar a falta de confiabilidade para estes alimentos no presente questionário. Além disso, acredita-se que os adolescentes apresentaram pouca familiaridade com a nomenclatura utilizada para o refrigerante light e a sopa industrializada. Para o consumo de creme de leite e batata cozida, observou-se dificuldade no relato da quantidade consumida, uma vez que estes alimentos geralmente são consumidos em preparações, como estrogonofe e salada de maionese.

Na análise da concordância da classificação em quartis do consumo bruto de energia e nutrientes observaram-se valores de kappa ponderado bruto significativos (média $=0,50$ ) e para o consumo de nutrientes ajustado pelo consumo de energia, observou-se que os valores de kappa foram menores, porém se mantiveram significativos (média $=0,46$ ). Hansson et al. 26 observaram valores de kappa ponderado similares quando analisaram a concordância do consumo de energia e nutrientes $(0,28$ a 0,61 ; mediana $=0,40)$ categorizado em quartis.

Quanto à concordância da classificação em um mesmo quartil, os dados observados (média = $41 \%)$ também foram comparáveis aos de Hansson et al. 26 (média $=45 \%$ ).

A confiabilidade de QFA para adolescentes no Brasil foi avaliada em estudo desenvolvido na cidade de São Paulo. Marchioni et al. 28 analisaram a reprodutibilidade de QFA previamente validado para adolescentes de escolas públicas da cidade de São Paulo. A confiabilidade do questionário foi testada entre 49 adolescentes (25 meninos e 24 meninas) com idades entre 16 e 19 anos e o intervalo de tempo entre o teste-reteste foi de três meses. Os autores observaram $54,2 \%$ de concordância média e valores de kappa ponderado variando de 0,31 a 0,56 (média $=0,42$ ) para a classificação em tercis, resultados comparáveis aos obtidos neste estudo.

Os coeficientes de correlação de Pearson encontrados neste estudo situam-se próximos aos valores que Willett \& Lenart 9 consideram razoáveis quando dados de consumo alimentar são comparados com informações de marcadores bioquímicos, ou seja, entre 0,5 a 0,7 . Entretanto, alguns autores têm criticado a utilização do coeficiente de correlação nas análises de reprodutibilidade e validade do QFA, uma vez que os coeficientes não medem a concordância entre duas administrações do questionário e sim o grau com que estas duas aplicações estão relacionadas 14,17,29. Dessa forma, a concordância entre as duas aplicações do questionário foi analisada também segundo método proposto por Bland \& Altman 17.

Poucos estudos têm analisado a reprodutibilidade de QFA aplicando o método de Bland \& Altman 17. Marchioni et al. 28 observaram que o consumo de energia e macronutrientes foi superestimado na primeira aplicação do QFA em comparação à segunda aplicação. Andersen et al. 30 verificaram a reprodutibilidade do QFA quantitativo entre 103 estudantes adolescentes e observaram que houve tendência de aumento das diferenças entre os consumos de energia e nutrientes estimados nas duas aplicações do questionário com o aumento do consumo para a maioria dos nutrientes analisados. Huybrechts et al. 31 analisaram a reprodutibilidade de QFA específico para estimar consumo de cálcio entre crianças pré-escolares na Bélgica e verificaram que diferença média do consumo de cálcio entre as duas aplicações do QFA foi 23,8mg por dia e que a estimativa do consumo de cálcio na segunda administração foi $3 \%$ menor em comparação com a primeira aplicação do método.

Observou-se que a concordância do consumo de vitamina A bruto e ajustado para energia variou segundo a magnitude do consumo. Contudo, a concordância da categorização em quartis do consumo de vitamina A foi de $85 \%$ (concordância exata e adjacente) e estimou-se correlação estatisticamente significativa e que pode ser considerada razoável em estudos de reprodutibilidade de QFA $(\mathrm{r}=0,52)$.

Uma possível limitação desse estudo é o fato de que a obtenção dos dados de base para a elaboração da lista de alimentos do QSFA pode não ter contemplado período suficientemente amplo para obter informações sobre o consumo de nutrientes que são provenientes de produtos sujeitos a variações sazonais, basicamente de frutas e hortaliças (vitaminas A e C, por exemplo).

$\mathrm{O}$ trabalho aqui descrito possui duas características que demarcam a sua contribuição para o conhecimento na área de investigação do consumo de alimentos no Brasil: (1) apresenta a reprodutibilidade do relato dos itens alimentares da lista do QSFA e (2) é o primeiro estudo que analisa a reprodutibilidade de questionário desenvolvido no Rio de Janeiro para adolescentes. O desenvolvimento deste QSFA continuará com a verificação da sua validade e a avaliação sob o ponto de vista da linguagem e da forma, tendo em vista torná-lo um instrumento autopreenchível. Além disso, está sendo elaborado um manual fotográfico para possibilitar estimativas mais precisas das porções consumidas. 


\section{Conclusão}

A concordância entre duas aplicações de QSFA para adolescentes foi analisada aplicando-se diferentes procedimentos estatísticos. $\mathrm{Na}$ análise de itens alimentares, os coeficientes de correlação intraclasse foram adequados para a avaliação do relato de consumo de alimentos, exceto para creme de leite, doce de leite, batata cozida, refrigerante light e sopa industrializada, para os quais, é necessário revisar a forma de questio- nar. Ademais, os coeficientes de correlação de Pearson e o kappa ponderado para o consumo de energia, macronutrientes e micronutrientes analisados foram significativos e demonstraram que o QSFA em análise é capaz de gerar relatos com reprodutibilidade aceitável. Excetuando a vitamina $A$, as concordâncias entre as duas aplicações do questionário foram independentes da magnitude do consumo, reafirmando que os resultados de confiabilidade do QSFA para os adolescentes foram adequados.

\section{Resumo}

A reprodutibilidade de questionário semiquantitativo de freqüência alimentar (QSFA) para adolescentes do Rio de Janeiro, Brasil, foi analisada entre 108 adolescentes. O intervalo médio entre as duas aplicações do QSFA foi de vinte dias. A lista de alimentos e as porções de referência do QSFA basearam-se em dados de registros alimentares de 430 adolescentes. Estimaramse coeficientes de correlação intraclasse para itens alimentares e coeficientes de correlação de Pearson para nutrientes. A concordância da classificação em quartis para nutrientes foi estimada pelo kappa ponderado. O método de Bland-Altman avaliou a concordância para nutrientes. Apresentaram-se dados brutos e ajustados pela energia. Para os itens alimentares, a média dos coeficientes de correlação intraclasse foi 0,54. Para dados ajustados, a concordância para o mesmo quartil e quartis adjacentes variou de $72 \%$ a $86 \%$. Segundo o método de Bland-Altman, a concordância média variou de $77 \%$ a $104 \%$ e foram independentes da magnitude do consumo, exceto para vitamina A. Os coeficientes de correlação de Pearson variaram de 0,40 a 0,61. O QSFA demonstrou confiabilidade aceitável para as estimativas de alimentos e nutrientes de adolescentes da área considerada.

\section{Colaboradores}

R. A. Pereira foi responsável pela concepção do projeto, elaboração do instrumento, coordenação do trabalho de coleta de dados, análise e interpretação dos dados, participou também da redação do manuscrito. M. C. Araújo participou na elaboração do instrumento e foi responsável pelo treinamento da equipe, coleta, análise e interpretação dos dados, também participou da redação do manuscrito. D. M. Ferreira contribuiu na análise e interpretação dos dados. Todas as autoras são responsáveis pela autoria e revisão final do texto.

\section{Agradecimentos}

À professora Gloria Valeria da Veiga por ter disponibilizado os dados de consumo alimentar que permitiram a elaboração do instrumento e por suas valiosas contribuições na análise dos dados. À professora Rosely Sichieri pelas enriquecedoras sugestões na concepção e condução do projeto e contribuições na análise dos dados. Ao Conselho Nacional de Desenvolvimento Científico e Tecnológico (CNPq; processo no. 506336/2004-2) pelo financiamento do projeto. 


\section{Referências}

1. Willett WC. Overview of nutritional epidemiology. In:WillettWC, editor. Nutritional epidemiology. 2nd Ed. Oxford: Oxford University Press; 1998. p. 3-17.

2. Beaton GH. Approaches to analysis of dietary data: relationship between planned analyses and choice of methodology. Am J Clin Nutr 1994; 59(1 Suppl):253S-61S.

3. Gibson RS. Measuring food consumption of individuals. In: Gibson RS, editor. Principles of nutritional assessment. 2nd Ed. Oxford: Oxford University Press; 2005. p. 41-64.

4. Fisberg RM, Martini LA, Slater B. Métodos de inquéritos alimentares. In: Fisberg RM, Slater B, Marchioni DML, Martini LA, organizadores. Inquéritos alimentares: métodos e bases científicos. São Paulo: Editora Manole; 2005. p. 1-29.

5. Nelson $\mathrm{M}$. The validation of dietary questionnaires. In: Margetts BM, Nelson M, editors. Design concepts in nutritional epidemiology. 2nd Ed. Oxford: Oxford University Press; 1997. p. 266-95.

6. Lopes C. Reprodutibilidade e validação de um questionário de freqüência alimentar [Tese de Doutorado]. Portugal: Universidade do Porto; 2000.

7. Slater B, Lima FEL. Validade e reprodutibilidade dos métodos de inquérito alimentar. In: Fisberg RM, Slater B, Marchioni DML, Martini LA, organizadores. Inquéritos alimentares: métodos e bases científicos. São Paulo: Editora Manole; 2005. p. 108-28.

8. Lopes ACS, Caiaffa WT, Mingoti SA, Lima-Costa MFF. Ingestão alimentar em estudos epidemiológicos. Rev Bras Epidemiol 2003; 6:209-19.

9. Willett WC, Lenart E. Reproducibility and validity of food-frequency questionnaire. In: Willett WC, editor. Nutritional epidemiology. 2nd Ed. Oxford: Oxford University Press; 1998. p. 101-47.

10. Block G, Hartman AM, Dresser CM, Carroll MD, Gannon J, Gardner L. A data-based approach to diet questionnaire design and testing. Am J Epidemiol 1986; 124:453-69.

11. Araújo MC, Melo LPC, Ribeiro RL. Enquete sobre utensílios mais utilizados para servir alimentos. In: Livro de resumos da XXVII Jornada Giulio Massarani de Iniciação Científica, Artística e Cultural da Universidade Federal do Rio de Janeiro. Rio de Janeiro: Universidade Federal do Rio de Janeiro; 2005. p. 304.

12. Sichieri R, Everhart JE. Validity of a Brazilian food frequency questionnaire against dietary recalls and estimated energy intake. Nutr Res 1998; 18:1649-59.

13. Field AE, Peterson KE, Gortmaker SL, Cheung L, Rockett H, Fox MK, et al. Reproducibility and validity of a food frequency questionnaire among fourth to seventh grade inner-city school children: implications of age and day-to-day variation in dietary intake. Public Health Nutr 1999; 2:293-300.

14. Cade J, Thompson R, Burley V, Warm D. Development, validation and utilization of food-frequency questionnaires: a review. Public Health Nutr 2002; 5:567-87.
15. Andrade RG, Pereira RA, Sichieri R. Consumo alimentar de adolescentes com e sem sobrepeso do Município do Rio de Janeiro. Cad Saúde Pública 2003; 19:1485-95.

16. Salles-Costa R, Pereira RA, Vasconcellos MTL, Veiga GV, Marins VMR, Jardim BC, et al. Associação entre fatores socioeconômicos e insegurança alimentar: estudo de base populacional na Região Metropolitana do Rio de Janeiro, Brasil. Rev Nutr 2008; 21 Suppl:99-109.

17. Bland JM, Altman DG. Statistical methods for assessing agreement between two methods of clinical measurement. Lancet 1986; 1:307-10.

18. Rockett HRH, Colditz GA. Assessing diets of children and adolescents. Am J Clin Nutr 1997; 65 (4 Suppl):1116S-22S.

19. Anding JD, Kubena KS, Mcintosh WA, O'Brien B. Blood lipids, cardiovascular fitness, obesity, and blood pressure: the presence of potential coronary heart disease risk factors in adolescents. J Am Diet Assoc 1996; 96:238-42.

20. Carroll RJ, Pee D, Freedman LS, Brown CC. Statistical design of calibration studies. Am J Clin Nutr 1997; 65(4 Suppl):1187S-9S.

21. Burley V, Cade J, Margetts B, Thompson R, Warm D. Consensus document on the development, validation and utilization of food frequency questionnaires. In: The Fourth International Conference on Dietary Assessment Methods. Leeds: Nuffield Centre for International Health and Development, Leeds Institute of Health Sciences, University of Leeds/Southampton: Institute of Human Nutrition, University of Southampton; 2000. p. 3-48

22. Matthys C, Pynaert I, Keyzer W, Henauw S. Validity and reproducibility of an adolescent web-based food frequency questionnaire. J Am Diet Assoc 2007; 107:605-10.

23. Pérez-Cueto Eulert EJ, Roberfroid D, Kolsteren PW. Desarrollo y evaluación de un cuestionario semicuantitativo de frecuencias alimenticias para adolescentes bolivianos. Nutr Hosp 2006; 21:573-80.

24. Cullen KW, Zakeri I. The youth/adolescent questionnaire has low validity and modest reliability among low-income African-American and Hispanic seventh- and eighth grade youth. J Am Diet Assoc 2004; 104:1415-9.

25. Robinson S, Skelton R, Barker M, Wilman C. Assessing the diet of adolescent girls in the UK. Public Health Nutr 1999; 2:571-7.

26. Hansson LM, Galanti MR, Bergström R. Factors affecting reproducibility of a dietary reports using food frequency questionnaires. Eur J Clin Nutr 2000; 54:685-64.

27. Speck BJ, Bradley CB, Harrell JS. A food frequency questionnaire for youth: psychometric analysis and summary of eating habits in adolescents. J Adolesc Health 2001; 28:16-25.

28. Marchioni DML, Voci SM, Lima FEL, Fisberg RM, Slater B. Reproducibility of a food frequency questionnaire for adolescents. Cad Saúde Pública 2007; 23:2187-96. 
29. Ambrosini GL, van Roosbroeck SA, Mackerras D, Fritschi L, Klerk NH, Musk AW. The reliability of ten-year dietary recall: implications for cancer research. J Nutr 2003; 133:2663-8.

30. Andersen LF, Nes M, Lillegaard IT, Sandstad B, Bjørneboe GE, Drevon CA. Evaluation of a quantitative food frequency questionnaire used in a group of Norwegian adolescents. Eur J Clin Nutr 1995; 49:543-54.
31. Huybrechts I, Bacquer DD, Matthys C. Validity and reproducibility of a semiquantitative food-frequency questionnaire for estimating calcium intake in Belgian preschool children. Br J Nutr 2006, 95:802-16.

Recebido em 02/Jan/2008

Versão final reapresentada em 28/Abr/2008

Aprovado em 06/Mai/2008 